\title{
Pauline SCHMITT PANTEL, Aithra et Pandora. Femmes, Genre et Cité dans la Grèce Classique
}

\author{
Violaine Sebillotte Cuchet
}

\section{(2) OpenEdition \\ Journals}

\section{Édition électronique}

URL : http://journals.openedition.org/clio/9500

DOI : 10.4000/clio.9500

ISSN : 1777-5299

Éditeur
Belin

Édition imprimée

Date de publication : 15 décembre 2009

Pagination : 261-264

ISSN : 1252-7017

\section{Référence électronique}

Violaine Sebillotte Cuchet, «Pauline schmitt pantel, Aithra et Pandora. Femmes, Genre et Cité dans la Grèce Classique », Clio. Histoire, femmes et sociétés [En ligne], 30 | 2009, mis en ligne le 27 janvier 2010, consulté le 21 septembre 2020. URL : http://journals.openedition.org/clio/9500 ; DOI : https://doi.org/ $10.4000 /$ clio. 9500

Ce document a été généré automatiquement le 21 septembre 2020.

Tous droits réservés 


\title{
Pauline SCHMITT PANTEL, Aithra et Pandora. Femmes, Genre et Cité dans la Grèce Classique
}

\author{
Violaine Sebillotte Cuchet
}

\section{RÉFÉRENCE}

Pauline schmitt PANTel, Aithra et Pandora. Femmes, Genre et Cité dans la Grèce Classique, Paris, L'Harmattan, Bibliothèque du féminisme, 2009,224 pages.

1 En choisissant de publier ce recueil d'articles (écrits et publiés entre 1977 et 2008) sur les femmes et le genre dans la collection dont Hélène Rouch fut l'une des fondatrices, Pauline Schmitt Pantel salue le rôle joué par la recherche féministe dans le renouvellement de l'historiographie de l'antiquité. Dans un avant-propos qui rappelle combien la recherche se nourrit de l'engagement de son auteur-e, elle dévoile discrètement quelques traces d'une autobiographie qui comporte, on s'en doute, ses propres héroïnes. L'introduction, en quelques courtes pages, rappelle le texte fameux qui avait ouvert l'atelier du Centre Louis-Gernet et était dédié à la femme sans nom d'Ischomaque (Mètis 9, 1994, p. 299-305). Il sonne comme une mise en garde : les « droits et pouvoirs des femmes " dans le monde grec se définissent dans la négation. Aucune des recherches actuelles sur les multiples arrangements des anciens avec le statut juridique des femmes ne saurait faire oublier le discours politique et normatif généralisé qui en fait des inférieures à vie liées à leur fonction procréative.

2 La première partie rappelle ensuite en deux étapes, 1982 (colloque de Saint-Maximin : «Une histoire des femmes est-elle possible?») et 1990 (première édition de l'Histoire des femmes en Occident), comment, nourrie de l'anthropologie et directement orientée dans une perspective politique, la réflexion de l'auteure s'est constituée, avec ou sans le mot, dans une perspective de genre. "La différence des sexes. Histoire, anthropologie et cité grecque » reste étonnamment d'actualité dans sa recommandation de réfléchir 
non sur les femmes pensées comme un groupe séparé, mais sur ce que les sociétés ont fait de la différence sexuelle. La seconde étape, en 1990, fait le point sur des notions, dont celle du genre, en montrant à quel point la recherche est dépendante dans ses questionnements des concepts de son époque.

3 La deuxième partie, sur les jeunes et la cité, traite surtout du mariage. La valeur sociale de celle que l'on épouse prend un aspect très politique dans l'analyse du rite en l'honneur d'Athéna Apatouria à Trézène. Exceptionnellement, Athéna, déesse de la cité, est invoquée par les jeunes filles (parthenoi) au moment de leur mariage. Par le don de leur ceinture, elles se placent sous la protection de la divinité rusée qui va les faire passer de l'étape enfantine à celle de la sexualité et de l'enfantement des citoyens. Aithra, première héroïne éponyme de l'ouvrage, constitue le paradigme de ces jeunes filles; princesse de Trézène, elle donne naissance à Thésée par son union avec le roi d'Athènes, Egée, ou, selon d'autres versions, avec le dieu Poséidon. Mais l'héroïsme d'Aithra (protégée d'Athéna) n'est que celui de la déesse, jamais celui de la princesse : la conclusion de l'auteure marque ainsi un écart fondamental entre les héroïnes et les héros de la ruse, tels les chasseurs noirs. Seuls ces derniers sont de vrais héros, dotés d'une étoffe qui leur appartient en propre. Le troisième article de ce chapitre évoque les deux manières différentes de concevoir les relations entre les sexes, la manière tyrannique et la manière civique, si l'on peut dire. Pour punir les habitants de la cité de Corcyre, le tyran de Corinthe décide d'envoyer tous les jeunes Corcyréens en Lydie afin qu'ils y soient réduits à l'état d'eunuque. Or les habitants de la cité de Samos ayant intercepté le convoi décident d'intégrer les jeunes gens dans un rituel symbolisant le mariage, une manière civique de les sauver. L'union matrimoniale ressort de ces pages comme particulièrement étrangère au monde tyrannique. Dans «L'âne, l'adultère et la cité », Pauline Schmitt Pantel interroge cette fois l'institution par ses échecs : on y voit comment se construisent à la fois le masculin et le féminin par des contre-modèles tournés en dérision.

4 La troisième partie regroupe des articles plus récents qui enquêtent sur les constructions de genre visibles dans des espaces spécifiques : ici le sanctuaire et la salle de banquet, deux domaines qui sont au cœur des recherches de l'auteure. Après les archéologues qui ont enquêté sur les espaces funéraires ainsi que sur la maison et son gynécée, elle met en question la césure, trop rapidement établie dans l'historiographie, entre gestes rituels féminins et gestes rituels masculins: laver la statue du dieu, la vêtir, la porter, prier, sont des actes accomplis par les deux sexes et si l'on s'en tient aux gestes; il n'y a rien qui oppose une piété féminine et une piété masculine. En ce qui concerne les banquets, la réflexion se porte d'abord sur la question des «festins de femmes » dont l'auteure interroge la dimension exceptionnelle. En s'arrêtant ensuite sur les images, elle observe combien est opératoire une autre césure que celle du sexe : femmes et jeunes (imberbes) font des gestes similaires et s'opposent pareillement aux hommes plus âgés (barbus) qui sont incontestablement dans une position statutaire supérieure, identifiée avec celle des citoyens.

5 La dernière partie rassemble des articles portant sur le thème de la violence et de l'héroïsme. On y trouve l'histoire des Danaïdes qui, sauf l'une d'elles, Hypermestre, tuent leurs époux le soir de leurs noces, et surtout l'histoire d'Atalante dont la course armée ressemble à une chasse à l'homme. Pauline Schmitt Pantel rappelle combien la violence féminine, excepté la folie furieuse, est rare dans le monde grec. La seule violence positive des femmes est celle qu'elles mettent en œuvre lorsqu'il s'agit de 
défendre l'ordre politique et sexuel de la filiation. Ces femmes-là, épouses, filles, ou mères dans la cité, sont les vraies héroïnes du monde grec. L'auteure renvoie ainsi, à juste titre, la question de l'héroïsme à ceux qui le fabriquent : comment une société où femmes et hommes ne sont pas égaux peut-elle penser l'héroïsme au féminin ? L'analyse du texte de Plutarque, Vertus de femmes, dont Pauline Schmitt Pantel donne un autre aperçu dans ce numéro de Clio, permet de souligner à quel point l'héroïsme féminin s'inscrit dans un cadre étroit, celui du salut de la maison, voire de la cité.

6 Pandora, sur laquelle se clôt le volume, est interprétée comme symbolisant la spécificité des Grecs dans leur construction du féminin : différente de l'Eve biblique, Pandora est pur charme, beauté et séduction, mais elle ne participe pas à l'humanité qui est celle des mâles. Pandora constitue donc une race à part, celles des oisives qui ne connaissent ni travail ni souffrance et ne font que fatiguer l'homme. Reflet d'une pensée misogyne qui serait celle des Grecs en général ou reflet d'une construction historique et historiographique dont la particularité historique n'a pas encore été démontrée ? La question se pose aujourd'hui quand des traditions culturelles parallèles, en Grèce même, donnent des indices d'héroïnes positives y compris en dehors du contexte de la maternité et de la fidélité civique. Il n'est pas certain en effet que le féminin traditionnellement valorisé et dont la dimension politique est très justement soulignée par l'auteure, ne demande pas aussi à être plus précisément contextualisé. À quelle conception des sexes renvoie-t-il donc?

7 Une telle question indique à quel point le lecteur d'aujourd'hui peut non seulement admirer la constance de la pensée de Pauline Schmitt Pantel, son actualité depuis 1977, mais surtout sa dimension toujours prospective. La logique de genre propre à la cité démocratique et classique, en gros il s'agit d'Athènes qui est le point de focalisation des sources de la tradition manuscrite, est ici très clairement et intelligemment présentée avec des articles qui donnent des aperçus cohérents sur des thématiques précises (mariage, tyrannie, héroïsme, etc). Il reste à enquêter sur les autres logiques de genre, celles pour lesquelles certains des articles plus récents de l'auteure apportent d'ailleurs déjà des éléments qui encourage à poursuivre la perspective ; ainsi sur les gestes au sanctuaire ou les rapports des individus au banquet, deux contextes dans lesquels la polarité homme/femme est moins signifiante que celle qui oppose non-citoyens et citoyens ou fidèles du dieu et individus indifférents au sanctuaire. C'est probablement dans cette troisième partie consacrée aux espaces que l'élan prospectif est le plus sensible: le lecteur en sort convaincu qu'il y a encore matière à travailler et à découvrir, à condition d'impliquer systématiquement une documentation de nature archéologique. C'est alors seulement que des logiques de genre contemporaines et différentes de celle que nous désignons comme spécifique du monde "classique », pour le dire de manière très générale et en reprenant l'adjectif du titre de ce livre, pourront être mises au jour et permettront de restituer une vision plus complète des relations sociales en Grèce ancienne. 